\title{
Computing the positioning error of an upper-arm robotic prosthesis from the observation of its wearer's posture.
}

\author{
Alexis Poignant, Mathilde Legrand, Nathanaël Jarrassé and Guillaume Morel
}

\begin{abstract}
When the arm prosthesis worn by an amputated Human being is not adequately configured with respect to the end-effector task, body compensations are often observed. Namely, to compensate for a wrong joint positioning on the robotic distal side, a subject trying to reach a desired position/orientation of his/her hand mobilizes his/her proximal joints, thus exploiting the redundancy of the human+robot kinematic chain.

In this paper, we explore the possibility of exploiting this well-known behavior to reverse the causality: if we observe the posture of an amputated subject wearing a prosthesis during a hand positioning task, to what extent can we infer the positioning error of the prosthesis?

To answer this question, we make the assumption that the adequate, or natural posture for a given task is one that optimizes a postural score. The proposed approach then consists in i) measuring the joint posture of the subject fitted with the prosthesis; ii) search for an alternative posture that optimizes a postural score within the null space of the human+robot kinematic chain and iii) compute the position error for the robot joints between the initial and the optimized posture.

An experimental evaluation is provided with non amputated subjects who emulate erratic positioning of their distal joints during hand positioning tasks. Results show that joint errors are estimated with a precision that seems compatible with the implementation of a real time control algorithm.
\end{abstract}

\section{BACKGROUND}

Mechanical achievements, such as the augmentation of the number of DoF and the weight reduction [1], have paved the way for a new generation of prosthetic arms, both more functional and comfortable. These electrical prosthesis should, with time, match the benefits of body-powered prosthesis (precision and design for heavy work [2]) while being more comfortable and providing a greater range of functions [3]).

Commercially available upper-limb electric prostheses are controlled by their wearer through voluntarily generated auxiliary signals, most often electromyograhic signals (EMG). Though, surface EMG measurement highly depends on skin surface factors [3], considerably reducing the control robustness, while requiring extended learning phases from users, especially when dealing with the control of multiple active DoF. Meanwhile, the use of auxiliary signals generates two parallel control loops for the users: the body natural sensorimotor close-loop and the auxiliary prosthetic open-loop. The management of these two parallel loops is complex and non-natural. Therefore, users tend to underuse the prosthetic

*This work was supported by ANR BYCEPS, ANR-18-CE19-0004.

All authors are with Sorbonne Université, CNRS, INSERM, Institute for Intelligent Systems and Robotics (ISIR), Paris, France name. surnamedsorbonne-universite.fr
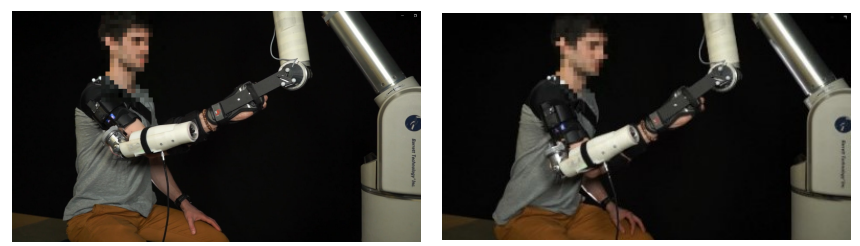

Fig. 1: A subject equipped with a robotized elbow, and having his wrist and hand locked, is asked to position a distal point at a given location materialized by a robot manipulator. On the left, a correct elbow positioning $\left(135^{\circ}\right.$ between the human humerus and the robotic ulna) results in a global posture that seems natural. On the right, with an erratic elbow joint position $\left(90^{\circ}\right.$ between humerus and ulna) the subject has to lean his trunk to achieve the task. This is called a body compensation.

joints to the benefit of residual functional joints with body compensations [1].

In the present paper, we aim at exploiting this natural behavior to evaluate whether a robotic prosthesis is correctly positioned during a manipulation task. The general idea, when looking at Fig. 1, consists in trying to compute the natural posture adopted on the left image from the measurement of the erratic posture adopted on the right image. Given a task, computing a natural posture, or, reciprocally, characterizing an ergonomically erratic posture, is a problem that is widely studied in the domain of industrial workstation optimization, and more specifically in the specific context of human-robot cooperation in the factories.

A first approach is based on dynamic simulation of a virtual manikin, using energy and forces based criteria mixed with task criteria, [4], [5], [6]. These methods have proven to be efficient but the transposition from the virtual to the real world strongly depends on the human model accuracy, leading to the identification of subject specific parameters for real-world applications [7]. Besides, these methods return trajectories, and relies on quadratic programming, requiring heavy computation power, as well as complex sensors (force sensors for example) that may difficulty be integrated to light and movable cobots or prostheses for real-world assessments.

A second approach relies on lighter kinematic models. This can be done while accounting for ergonomic criteria [8] and task specific criteria [9] to continuously control the behavior of the cobot. These methods optimize the global RULA-score (which evaluates the exposure to risk factors associated with work related upper limb disorders [10]), either discretely [8] - and therefore controlling sequentially the postural objective - or by continuously fitting polynomial functions, requiring a large set of pre-recorded postures to do 
such interpolation [11]. Further, these approaches propose to correct the user posture by moving the manipulated tool or instrument in the task space. Rather, in a prosthetic approach, there is no possible control on the task. In fact, the task is a priori unknown. For this reason, we propose to assume that the task is correctly realized by the user thanks to compensation, and therefore to search for a possibly better joint configuration inside the null-space of the redundant human+prosthesis kinematic chain.

To this aim, generalized weighted inverse [12][13] can be used to link the end-effector velocity to the adequate prosthesis' motion. These models are computationally efficient but their weights are calculated by minimizing a criteria on previously recorded data, which requires important dataset from prosthesis users. These weights are also task specific, yet could be extended to multiple tasks by adding neural networks for classification [12], [14] but, again, the required dataset increases. Moreover, the weights do not ensure that the prosthesis movement will be ergonomically adequate, and the generalization from one user to another is complex.

To use ergonomic criteria without modifying the nullspace, we can exploit a projector [15], [16]. Commonly employed for the control of redundant robotic arms with a high number of DoF, null-space control can here be transposed to human + prosthesis kinematic model. Instead of looking for the appropriate weights to minimize a criteria on recorded data, the null-space allows to minimize an ergonomic criteria projected on the solution space. Moreover, this criteria is neither task specific nor user specific, making its extrapolation easier from one user to another without any records. This is why this approach was chosen.

The rest of the paper is organized as follows. We first detail the proposed optimization approach (Section II). Then we present the experimental protocol and setup that was used to gather data in order to evaluate the performance of our method (Section III). Experimental results are then provided (Section IV) and discussed (section V).

\section{PROPOSED APPROACH}

We parameterize the human+prosthesis kinematic chain by a joint angular position : $q=\left(q_{i}\right), i \in\{1 \cdots n\}$ with $n$ representing the total number of DoFs, including those of the prosthesis and those used to model the human kinematic chain. We suppose that a sensory device provides a measure $q_{m}$ of the joint posture $q$ at any time $t$.

We aim at computing $\hat{q}$, an optimized joint vector that results in the same end-effector position and orientation as $q_{m}$, while minimizing a cost function $C$. Inspiring from ergonomic scores such as RULA table, widely used to evaluate the goodness of a given posture defined by joint angles, [17], we consider that the optimal joint position corresponds to an upper body at rest, characterized by a desired position $q_{d}$.

The computed optimal position is defined by:

$$
\hat{q}=\underset{q}{\operatorname{argmin}} C\left(q, q_{d}\right) \text { s.t } f(q)=f\left(q_{m}\right)
$$

where $f($.$) denotes the kinematic model of the hu-$ man+prosthesis chain, mapping the joint position $q$ into the the hand position and orientation. This problem is iteratively solved by computing a null-space reconfiguration:

$$
\begin{aligned}
& q \leftarrow q_{\text {init }} \\
& \delta \leftarrow 2 \varepsilon \\
& \text { while } \delta>\varepsilon \text {, } \\
& \delta \leftarrow J^{\dagger}(q)\left[f\left(q_{m}\right)-f(q)\right] \\
& \begin{array}{l}
\left.\quad-\left[I-J^{\dagger}(q) J(q)\right)\right] \operatorname{grad}\left(C\left(q, q_{d}\right)\right) \\
q \leftarrow q+\delta
\end{array} \\
& \text { end while } \\
& \hat{q} \leftarrow q
\end{aligned}
$$

where $J(q) \in \mathbb{R}^{n \times 6}$ denotes the Jacobian matrix for a given joint position angle $q$; $\dagger$ the generalized inverse operator.

The step $\delta$ is formed of two terms: the task-space step $J(q)^{\dagger}\left[f\left(q_{m}\right)-f(q)\right]$, which ensures the respect of the constraint $f\left(q_{m}\right)=f(q)$ and the null-space step $\left.\left[I-J^{\dagger}(q) J(q)\right)\right] \operatorname{grad}\left(C\left(q, q_{d}\right)\right)$ that locally minimizes the cost function $C$. The convergence is obtained when the step $\delta$ is as small as a tunable positive threshold $\varepsilon$.

Finally, the postural error, between the natural and optimised model, is computed as the difference between $q_{m}$ and $\hat{q}$ the joint angle at convergence.

For the cost function $C$ we chose:

$$
C\left(q-q_{d}\right)=M\left\|Z\left(q-q_{d}\right)\right\|_{2}^{2},
$$

where $M \in \mathbb{R}_{+}^{n \times n}$ is a diagonal matrix of positive weights and $Z$ denotes a dead zone along each component of $q$, i.e.,

$$
Z_{i}\left(q-q_{d}\right)= \begin{cases}q_{i}-q_{d, i}+z_{i} & \text { if } q_{i}-q_{d, i}>+z_{i} \\ q_{i}-q_{d, i}-z_{i} & \text { if } q_{i}-q_{d, i}<-z_{i} \\ 0 \text { otherwise } & \end{cases}
$$

Therefore the $i$-th component of the gradient function $g=$ $\operatorname{grad}(C)$ writes:

$$
g_{i}\left(q-q_{d}\right)= \begin{cases}2 M_{i}\left(q_{i}-q_{d, i}+z_{i}\right) & \text { if } q_{i}-q_{d, i}>+z_{i} \\ 2 M_{i}\left(q_{i}-q_{d, i}-z_{i}\right) & \text { if } q_{i}-q_{d, i}<-z_{i} \\ 0 \text { otherwise } & \end{cases}
$$

\section{MAterial AND Methods}

\section{A. Kinematic model}

We consider the theoretical case of a person amputated at the humerus level, equipped with a 2 DoF prosthesis: elbow flexion + wrist prono-supination. The person is sat on a stool (thus legs are not involved in body compensations at this stage). The task consists in positioning/orienting the hand at a desired location.

The global kinematic model involves 9 revolute joints :

- 7 human actuated joints (consisting in a 3 DoF spherical pelvis joint, a 1 DoF scapula elevation joint and a 3 DoF spherical shoulder joint;

- 2 robotic joints (1 DoF elbow and a 1 DoF wrist).

The Dennavit and Hartenberg parameters of this model are described in Table I, where the length of the skeleton bones 
TABLE I: Denavit-Hartenberg parameters of the torso and right arm kinematic model

\begin{tabular}{|c|c|c|c|c|c|c|c|c|c|}
\hline \multicolumn{10}{|c|}{ Denavit-Hartenberg parameters } \\
\hline Joint & Trunk $_{1}$ & Trunk $_{2}$ & Trunk $_{3}$ & Scapula & Shoulder $_{1}$ & Shoulder $_{2}$ & Shoulder $_{3}$ & Elbow $^{\text {Wrist }}$ \\
\hline$\alpha$ & $\pi / 2$ & $-\pi / 2$ & $-\pi / 2$ & 0 & $\pi / 2$ & $-\pi / 2$ & $\pi / 2$ & $\pi / 2$ & 0 \\
\hline$\theta_{\text {offset }}$ & 0 & 0 & 0 & 0 & 0 & $-\pi / 2$ & 0 & $\pi$ & 0 \\
\hline $\mathrm{a}$ & 0 & 0 & 0 & $l_{\text {scapula }}$ & 0 & 0 & 0 & 0 & 0 \\
\hline $\mathrm{d}$ & 0 & 0 & $l_{\text {torso }}$ & 0 & 0 & 0 & $l_{a}$ & 0 & $l_{f a}$ \\
\hline
\end{tabular}

are noted $l_{\text {torso }}, l_{\text {scapula }}, l_{a}, l_{f a}$, for respectively the torso, the scapulae, the arm and the forearm.

Such a kinematic model is the one provided by the skeleton tracking functionality of a Microsoft Kinect V2 sensor which is used to measure $q_{m}$. Figure 2 shows a photo of a subject observed by the Kinect together with the superimposed extracted skeleton.

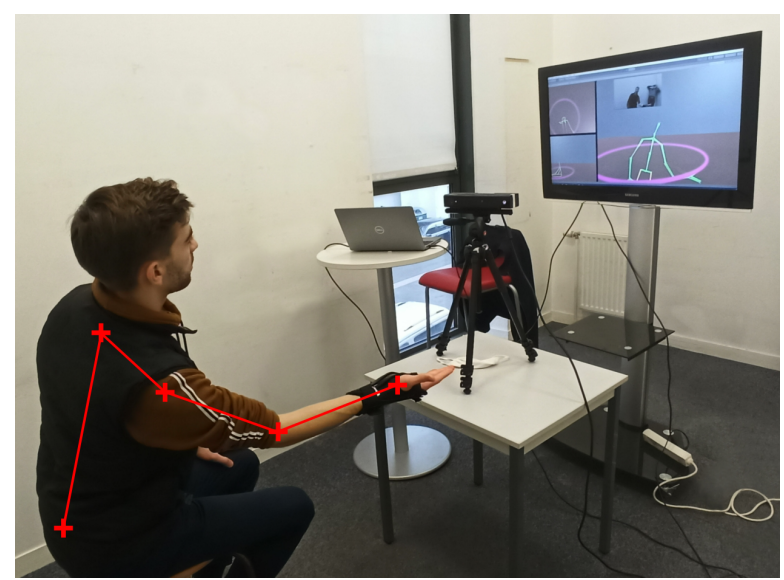

Fig. 2: View of the experimental setup used: a subject (with his measured skeleton added in red), performing a compensatory movement (while maintaining his hand position and orientation) in front of the Kinect V2; A visual interface indicates the hand position/orientation to reach and displays the subject's skeletal model, from different perspectives

\section{B. RULA-inspired criteria}

Since the RULA score is a discrete score, it cannot be directly used as an optimization criteria. We rather use the ergonomically ideal joint angles to define a continuous cost function. Some joints, such as the trunk, are defined with the ideal position of $0^{\circ}$, whereas some joints are defined ergonomically good when within a given range. For example, the elbow is ideally position when its angle fits within $80^{\circ} \pm$ $20^{\circ}$. The ideal ergonomic posture is thus defined by the joint vector $q_{d}$ with associated deadzone vector $z$.

This criteria relies on the hypothesis that compensatory movements always degrade ergonomics, and that the desired posture $q_{d}$ remains the same, no matter the performed task.

To avoid having a cost function being weighted the same for all joints (which would make the trunk as ergonomically significant as the shoulder), the weighting matrix $M$ was designed in order to reflect the specificity of the RULA score for the different joints. Those weights were adjusted the following way: the higher a joint angle denotes a compensatory movement, the higher the associated weight should be, while a purely functional joint is not weighted.

TABLE II: Chosen optimization weights

\begin{tabular}{|l||l|l|l|l|l|l|}
\hline \multicolumn{7}{|c|}{ Optimization weights for each joint } \\
\hline Joint & Trunk $_{1,3}$ & Trunk $_{2}$ & Scapula & Shoulder $_{1,2,3}$ & Elbow & Wrist \\
\hline$M$ & 100 & 300 & 100 & 0 & 10 & 0 \\
\hline
\end{tabular}

In order to avoid over-fitting, the weights were tuned by hand (Table II). The shoulder and wrist weights are set to $0{ }^{\circ}$ as they are purely functional joints during the performed tasks. The elbow being highly functional (closely related to the distance of the target if the trunk is straight), its weight is also low.

More complex criteria can be derived from discrete scores, for example by fitting polynomial functions on sets of postures [9] but this requires to learn from a dataset of postures, which is difficult to obtain especially for specific population such as prosthesis users. Rather, the chosen cost function can be easily configured with a very small dataset.

\section{Protocol}

An experiment was conducted on 15 non-amputated subjects from 20 to 55 years old, from 1.60 to $1.90 \mathrm{~m}$ tall, 6 women and 9 men. Those had to perform hand positioning tasks at a given location. The positioning was first done in a natural way (without constraints). Second, subjects were asked to adopt an erratic positioning of their distal joints so as to mimic the case of an amputated subject with a prosthesis exhibiting an erroneous posture, and to reach the same given location for the hand. They were sat on a stool in order to limit the displacement of the pelvis and to compare more accurately the different posture with and without compensation. They were wearing a wrist splint to block their wrist flexion and abduction, to mimic a 2 DOF prosthesis configuration. Each subject performed 18 reaching tasks with their right arm, with and without (provoked) compensations. The 18 tasks consist of 6 positions: 2 distances $(80 \%$ and $60 \%$ maximum reach) $\times 3$ horizontal positions (in front of the right shoulder position and \pm the shoulder width $) \times 1$ height ( sternum height), across 3 different hand orientation: palm vertical, palm horizontal, palm at a $45^{\circ}$ to the ground. These 18 couples position/orientation represents 18 targets normalized to the subjects' size.

Each task goes as follow: I) The subjects are asked to reach the target naturally, and this "Natural Posture" is recorded. Then, while maintaining hand position and orientation, they are instructed to move their distal joints (wrist and elbow) according to pseudo-randomly drawn instructions : flexion 
or extension of the elbow and pronation or supination of the wrist (4 total compensations, each being accounting for $25 \%$ of the total compensated posture set). II) While keeping their distal joints blocked, they were asked again to reach the target. To do so, a visual feedback is provided on a screen. A "Compensated Posture" is then recorded. III) Offline, the optimization algorithm is run and return an "Optimal Posture". The total dataset is constituted of $15 * 18=270$ sets of postures, each set being composed of 1 natural posture (Nat), 1 compensated posture (Comp), and 1 optimal posture (Opt) returned by the algorithm.

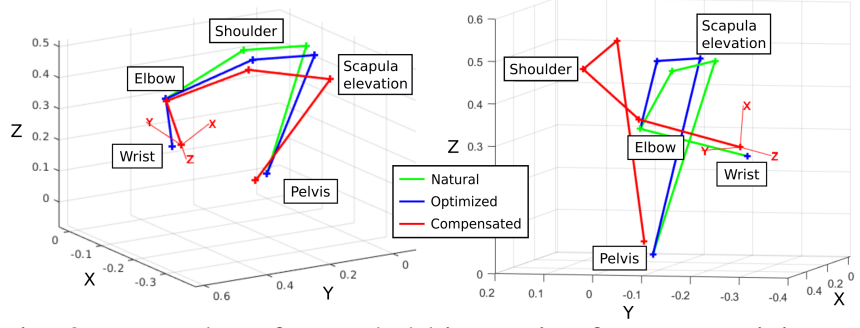

Fig. 3: Examples of recorded kinematics for one participant reaching two different targets and seen from two different angles. On the left the subject compensated by bending the trunk forward, while on the right, he compensated backward (because of imposed elbow extension). The computed optimized postures appear to be close to the natural ones in both cases. The data is centered on the pelvis' natural posture.

\section{Joint offset and jitter from the Kinect sensor}

The Kinect v2 Camera's joint detection faces important offsets and jitter [18][19]. In our protocol, we estimated, at 5 feet, the maximum jitter to be about $2.5 \mathrm{~cm}$. Consequently, we estimated the elbow angle's noise to be around $\pm 5^{\circ}$ for a $35 \mathrm{~cm}$ elbow-hand bone.

Similarly, the measure of the wrist pronosupination might suffer of equivalent noise, as well as the fact that subjects, between the compensated and natural posture, do not exactly orient their hand the same way; to solve this, the end-effector constraint $f_{q_{m}}$ is set as the measurement of the natural posture. The same observation occurs for the bone's length that varies between measures; therefore, during optimization, it is set as the natural posture lengths. And, finally, the base of the kinematic model being the position of the pelvic, for the same reason, we set the base of the optimized model to be the base of the natural kinematic model.

Joint offsets are strongly undesired for the pelvic joint which defines the base of our kinematic model, and strongly impact the back's leaning angle, which is the most weighted criteria of our optimization. In sitting position, the pelvis measured position is always overshot by a few centimeters, and consequently the trunk angle is over-estimated. Even when leaning backwards, the trunk angle is almost always positive. To compensate that offset, we do not set the trunk leaning angle desired $q_{d, 2}$ at $0^{\circ}$ but it is instead set as the mean angle of the natural posture for each subject (out of the 18 natural postures). That mean trunk angle $\overline{q_{n a t, T}}$ is, depending on the subject, between 5 and $18^{\circ}$. The same thing is performed for the scapula angle $\overline{q_{\text {nat }, B}}$ as the natural scapula elevation detected by the Kinect strongly depends on the subject physiognomy and clothing. The desired angles and the dead zones $Z$ chosen are summed up in Table III

TABLE III: Chosen Desired angle in ${ }^{\circ}$

\begin{tabular}{|l||l|l|l|l|}
\hline \multicolumn{5}{|c|}{ Desired angles for each joint } \\
\hline Joint & Trunk $_{1,3}$ & Trunk $_{2}$ & Scapula & Elbow \\
\hline$q_{d}$ & 0 & $\bar{q}_{\text {nat }, T}$ & $\overline{q_{\text {nat }, B}}$ & $80^{\circ}$ \\
\hline$Z$ & 0 & 0 & 0 & $\pm 20^{\circ}$ \\
\hline
\end{tabular}

\section{EXPERIMENTAL RESULTS}

\section{A. Postural errors between conditions}

The RMSE of the absolute errors between the "Natural" and "Compensated" measured postures (Nat-Comp) and between the "Natural" and the computed "Optimum" postures (Nat-Opt) are shown in the Table IV, which indicates that, globally, the Optimum postures are closer to the Natural ones, than the Compensated ones do.

TABLE IV: RMSE of the absolute errors

\begin{tabular}{|l||l|l|l|l|}
\hline \multicolumn{1}{|c||}{} & \multicolumn{4}{c|}{ RMSE of the absolute errors in ${ }^{\circ}$} \\
\hline Joint & Trunk 2 & Scapula & Elbow & Wrist \\
\hline Nat-Comp & 10.3354 & 8.4725 & 26.4834 & 12.8714 \\
\hline Nat-Opt & 2.6812 & 3.7261 & 4.6764 & 3.4311 \\
\hline
\end{tabular}

\section{B. Distribution of errors}

Figure 4 shows the histograms of errors, comparing the error between the "Natural" and "Compensated" measured postures (in orange) and the one between the "Natural" and the computed "Optimum" postures (in blue) for the trunk, scapula elevation, elbow joint angle and wrist joint angle. The histogram indicates that the distribution of the errors between Natural and Optimum postures are unimodal, centered on zeros and with a limited spread, clearly showing that the Optimum computed postures are closer to the Natural ones than the compensated ones. Looking at the Natural-Compensated error (orange), it can be noticed that for the elbow, the distribution looks like the sum of two Gaussian distributions, one representing the flexion and one the extension of the elbow that the experimenter asked the subjects to perform. Similar observations should be seen for the wrist, with pronation and supination, but with a flatter distribution as subjects tend to unintentionally move their wrist while trying to reach the targets. This also explains why no errors were sometimes observed between Natural and Compensated postures while a compensatory movement was asked. Finally, the trunk distribution shows that subjects prefer to lean forward than backward.

\section{Relative evaluation of errors}

Figure 5 presents the error between Natural and Optimum postures (Opt-Nat) as a function of the error between Natural and Compensated (Comp-Nat) ones. Oppositely to the histograms, these figures show paired input and output errors 

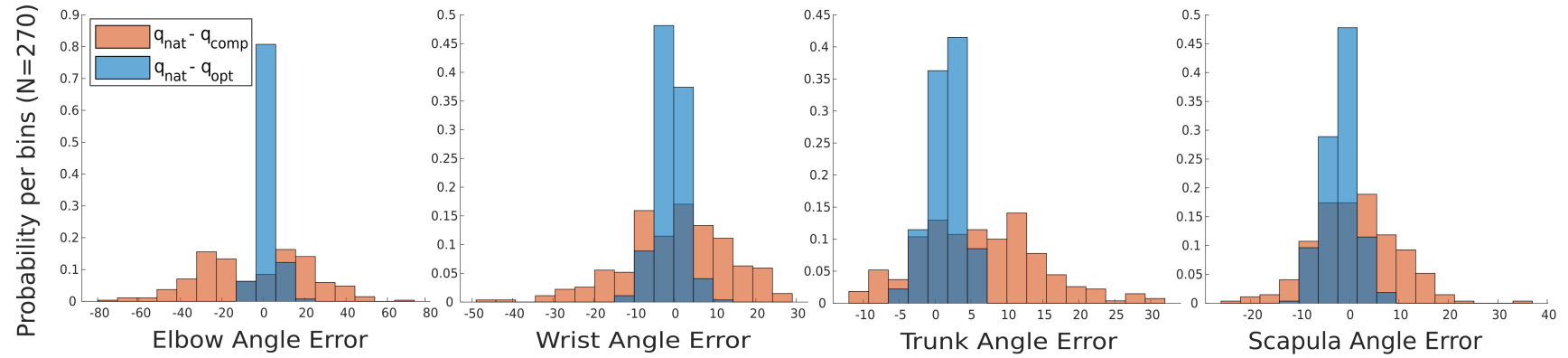

Fig. 4: From left to right: Histograms of the elbow, wrist, trunk and scapula angle error, before optimization (in orange, the error between natural and compensated) and after optimization (in blue, the error between natural and optimum)

of the optimisation, as we want each posture to be improved, and not just a general improvement of the mean error. On the proposed figure, a postural improvement for a given posture is defined by a point placed below the axis defined by $y=x$ (dashed black lines). The green zones denotes an improvement of the joint position for the given joint. The red zone denotes a deterioration. We also plotted margins of $\pm 5^{\circ}$ (black lines) that represent the estimated noise due to the Kinect. Therefore, points inside these margins may be considered as neither improved nor deteriorated. Finally, Figure 6 presents the same data but computed for the sum of the absolute errors of the 4 considered angles : trunk, scapula, elbow and wrist. This figure can be seen as a global classifier for detecting compensatory motions. Indeed, when a subject does not compensate, the Natural-Compensated and Natural-Optimised errors are equal and close to $0^{\circ}$ (less than 5 or $10^{\circ}$ for example, depending on the chosen threshold), while otherwise there is an important difference.

Out of the 270 postures, only 4 postures were misclassified (in the red zone Figure 5. for the elbow. These misclassified points have a null error Natural-Compensated regarding the elbow angle, meaning the algorithm identified high compensations on the other angles and cancelled them while increasing the elbow error. While only 2 postures were globally misclassified with a $5^{\circ}$ threshold (Figure 6), these postures have a global error Natural-Compensated close to $0^{\circ}$, meaning the subject actually did not compensated while asked. Finally, it can be seen that every postures with a global compensated-natural error of more than $20^{\circ}$ were correctly classified, meaning the global detection of compensatory motions was effective.

\section{DISCUSSION}

The experimental results indicates that the proposed nullspace optimization approach with its RULA-inspired score was able, for a given task (i.e. hand positioning and orienting), to compute, from a typical erroneous posture, a postural solution close to the natural and comfortable strategy exhibited by users. For all angles, the global RMSE was highly improved comparing before and after optimization. The method appeared to be effective on every tested posture and not only with a limited set of them as shown in Fig. 5.

\section{A. Extension to more complex models}

This results were obtained with a 9 DoF kinematic model, and a 6 DoF task, thus within a 3 DoF redundancy problem to optimize. As seen, we used important weights for the 3 DoF pelvic joint, and, in this configuration, converging to the 3 correct trunk angles should be sufficient to have a null error between the optimum and the natural position. This means that, in this specific case, there is only one viable strategy to reach the target without compensations. If we were to increase the complexity of the kinematic model with more DoF, the choice of the weight would be more critical to converge towards the natural posture within a larger set of solutions. For example, the weights of the optimization cost-function should probably be tuned more carefully for a 4-DoF prosthesis (with a spherical wrist), as the redundancy of the kinematic model would require 5 angles to be correctly estimated through optimization instead of 3 (for example, the flexion and abduction of the wrist should be close to $0^{\circ}$ ) but we think that this method could correctly be implemented on a 4-DoF prosthesis.

\section{B. Kinect limitations}

It should also be mentioned that optimum that is computed strongly depends on the end-effector position and orientation measured $f\left(q_{m}\right)$. The noise of the wrist, palm and thumb position strongly impacting $f\left(q_{m}\right)$ and therefore considering the global convergence for a spherical wrist, the Kinect might not be precise enough. However, for a $1 \mathrm{DoF}$ wrist model, the Kinect has proven to be precise enough in our experiment. Similarly, it is important to highlight that the Kinect noise affects the measured lengths of the bones from one posture to another (as previously mentioned in II.E). These lengths variations impact the determined joint position $q$. The natural and optimized joint positions are calculated for the same bone lengths, while the bone lengths of the non-natural posture vary by a couple of centimeters. Consequently, because of such difference, the error improvement should be nuanced. However, the results still remain significant and the visual postural improvement of the kinematic model is flagrant, as shown in Figure 3.

Nevertheless, RGB-D cameras might be viable for medical rehabilitation applications, as it is quite cheap and could 

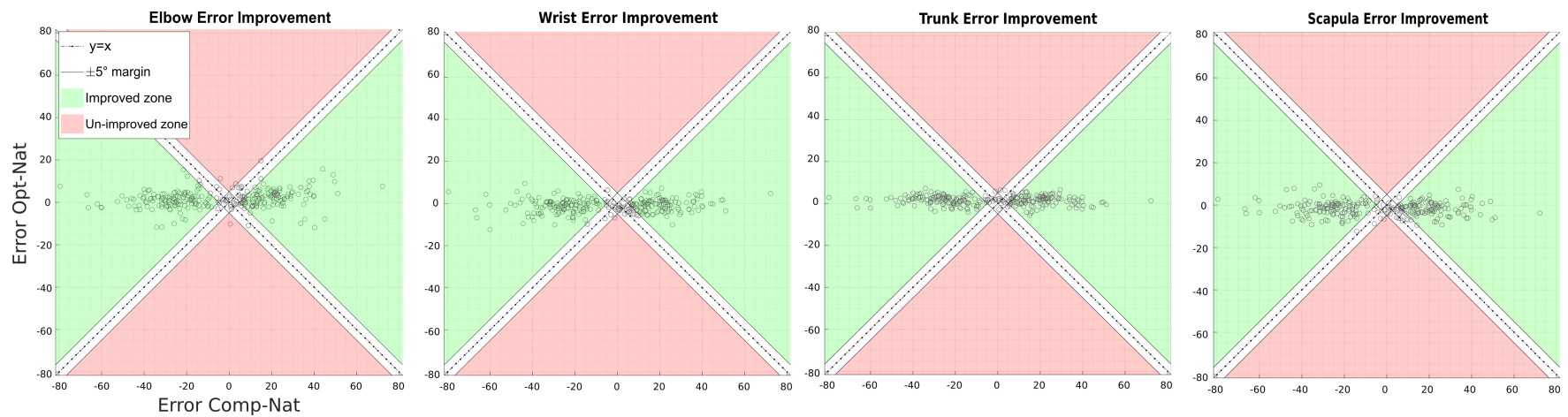

Fig. 5: The relative error (from left to right and top to bottom: elbow, wrist, trunk, scapula) after optimization as a function of the error before optimization. The dark dashed-dotted line represents the $y=x$ and $y=-x$ axis, and the thin black lines the $\pm 5^{\circ}$ margin of our estimated noise. An improve point prediction is located in the green zone, between the $y=x$ and $y=-x$ axis. The closer it is to the horizontal axis, the better the prediction is.

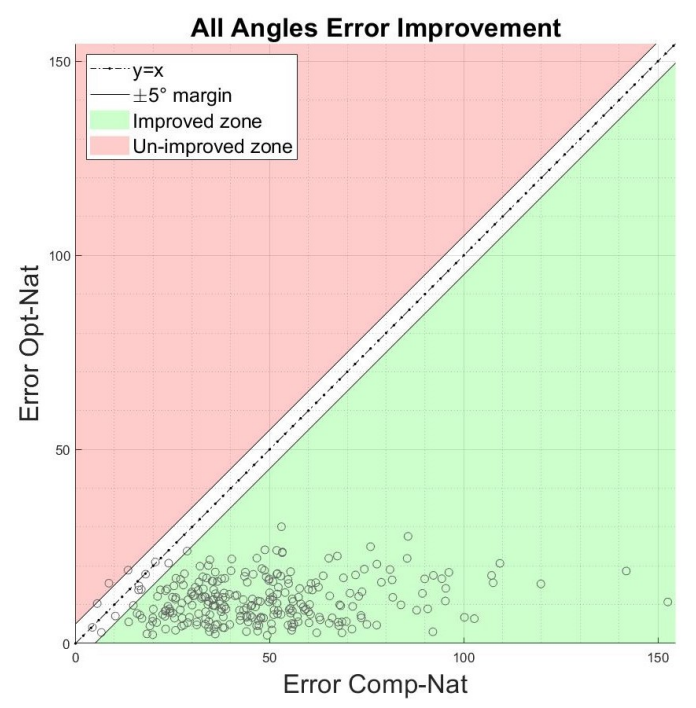

Fig. 6: Absolute error after optimization as a function of the error before optimization for the sum of the trunk, scapula, elbow and wrist angle. The dark dashed-dotted line represents the $y=x$ axis, and the thin black lines the $\pm 5^{\circ}$ margin of our estimated noise.

easily be set at patients' home, as well as industrial context in front of a worker's bench. The Kinect noise might even be reduced for real-time applications by using an Extended Kalman Filter [18]. However, for daily-life prosthetic implementations, the algorithm should be transposed to alternative technologies, such as Inertial Measurement Units to estimate the subjects postures for a use outside of a laboratory environment.

\section{Computational power}

The optimization algorithm being linear, it does not require high computational power: on a generic Intel core i5 laptop running non-optimized Matlab code, the optimization timing was about $10 \mathrm{~ms}$ (30 iterations) for a $0.1^{\circ}$ of precision error set as stopping criteria $\varepsilon$. Additionally in a real-time application to a prosthesis control such as in [20], since the optimization initial guess would be set to the posture obtained for the previous computation step, the error to cancel would be reduced and so would be the number of optimization iterations.

\section{CONCLUSION}

In this study we presented a simple approach to search for an alternative posture that optimizes an ergonomic postural score within the null space of a human kinematic chain. The results of a preliminary experimental campaign on a group of non-amputated subjects (emulating compensatory movements similar to the ones exhibited by amputated users with inadequate prosthesis position) indicates that this method is effective and able to precisely determine more ergonomic and natural solutions for the distal joints (wrist and elbow) angles as well as for the trunk and scapula. Unlike existing approaches, this was achieved with a simple extension of a discrete ergonomic score which is not task nor user specific, do not require any training data, and is computationally much more efficient that dynamic manikin based simulations as well as easier to parameterize.

While further work has to be conducted for this method to be implemented in realistic scenarios exploiting wearable motion sensors to reconstruct postural data, the proposed approach paves the way for multiple DoF prosthesis control. Indeed, it could be used as a joint error computation stage within a movement-based compensation cancellation control approach described in [20]. Further, this method could also be used to quantify compensatory motions in industrial or medical context, possibly extending existing discrete ergonomic scores.

\section{ACKNOWLEDGMENT}

The authors would like to thank all the participants of the experiment. This work was carried out in accordance with the recommendations of the University Paris Descartes ethic committee CERES.

\section{REFERENCES}

[1] F. Cordella, A. L. Ciancio, R. Sacchetti, A. Davalli, A. G. Cutti, E. Guglielmelli, and L. Zollo, "Literature review on needs of upper limb prosthesis users," Frontiers in neuroscience, vol. 10, p. 209, 2016. 
[2] S. Millstein, H. Heger, and G. Hunter, "Prosthetic use in adult upper limb amputees: a comparison of the body powered and electrically powered prostheses," Prosthetics and orthotics international, vol. 10, no. 1, pp. 27-34, 1986.

[3] D. Farina, N. Jiang, H. Rehbaum, A. Holobar, B. Graimann, H. Dietl, and O. C. Aszmann, "The extraction of neural information from the surface emg for the control of upper-limb prostheses: emerging avenues and challenges," IEEE Transactions on Neural Systems and Rehabilitation Engineering, vol. 22, no. 4, pp. 797-809, 2014.

[4] P. Maurice, Y. Measson, V. Padois, and P. Bidaud, "Assessment of physical exposure to musculoskeletal risks in collaborative robotics using dynamic simulation," in Romansy 19 - Robot Design, Dynamics and Control, V. Padois, P. Bidaud, and O. Khatib, Eds. Springer Vienna, 2013, vol. 544, pp. 325-332, series Title: CISM International Centre for Mechanical Sciences. [Online]. Available: http://link.springer.com/10.1007/978-3-7091-1379-0_40

[5] G. De Magistris, A. Micaelli, C. Andriot, J. Savin, and J. Marsot, "Dynamic virtual manikin control design for the assessment of the workstation ergonomy," in First International Symposium on Digital Human Modeling. Lyon, 2011.

[6] F. Ore, L. Hanson, N. Delfs, and M. Wiktorsson, "Virtual evaluation of industrial human-robot cooperation: An automotive case study," in 3rd International Digital Human Modeling Symposium (DHM2014), May 20-22, Odaiba, Japan, 2014.

[7] L. Peternel, W. Kim, J. Babic, and A. Ajoudani, "Towards ergonomic control of human-robot co-manipulation and handover," in 2017 IEEE-RAS 17th International Conference on Humanoid Robotics (Humanoids). IEEE, 2017, pp. 55-60. [Online]. Available: http://ieeexplore.ieee.org/document/8239537/

[8] A. Shafti, A. Ataka, B. U. Lazpita, A. Shiva, H. Wurdemann, and K. Althoefer, "Real-time robot-assisted ergonomics*," in 2019 International Conference on Robotics and Automation (ICRA). IEEE, 2019, pp. 1975-1981. [Online]. Available: https://ieeexplore.ieee.org/ document/8793739/

[9] B. Busch, G. Maeda, Y. Mollard, M. Demangeat, and M. Lopes, "Postural optimization for an ergonomic human-robot interaction," in 2017 IEEE/RSJ International Conference on Intelligent Robots and Systems (IROS). IEEE, 2017, pp. 2778-2785. [Online]. Available: http://ieeexplore.ieee.org/document/8206107/

[10] L. McAtamney and E. N. Corlett, "RULA: a survey method for the investigation of world-related upper limb disorders," p. 10, 1993.

[11] B. Busch, M. Toussaint, and M. Lopes, "Planning ergonomic sequences of actions in human-robot interaction," in 2018 IEEE International Conference on Robotics and Automation (ICRA), 2018, pp. 1916-1923.

[12] D. J. Lura, "The creation of a robotics based human upper body model for predictive simulation of prostheses performance," 2012.

[13] D. Menychtas, S. L. Carey, R. Alqasemi, and R. V. Dubey, "Upper limb motion simulation algorithm for prosthesis prescription and training "," in 2019 IEEE/RSJ International Conference on Intelligent Robots and Systems (IROS). IEEE, 2019, pp. 6495-6501. [Online]. Available: https://ieeexplore.ieee.org/document/8967658/

[14] Y. Xia and J. Wang, "A dual neural network for kinematic control of redundant robot manipulators," IEEE Transactions on Systems, Man, and Cybernetics, Part B (Cybernetics), vol. 31, no. 1, pp. 147-154, 2001.

[15] A. Liegeois et al., "Automatic supervisory control of the configuration and behavior of multibody mechanisms," IEEE transactions on systems, man, and cybernetics, vol. 7, no. 12, pp. 868-871, 1977.

[16] B. Siciliano, "Kinematic control of redundant robot manipulators: A tutorial," Journal of intelligent and robotic systems, vol. 3, no. 3, pp. 201-212, 1990.

[17] Ergo-Plus. (2020) A step-by-step guide to the rula assessment tool. [Online]. Available: https://ergo-plus.com/wp-content/uploads/ rapid-upper-limb-assessment-rula-1.png

[18] J. Shu, F. Hamano, and J. Angus, "Application of extended kalman filter for improving the accuracy and smoothness of kinect skeletonjoint estimates," Journal of Engineering Mathematics, vol. 88, no. 1, pp. 161-175, 2014.

[19] Q. Wang, G. Kurillo, F. Ofli, and R. Bajcsy, "Evaluation of pose tracking accuracy in the first and second generations of microsoft kinect," in 2015 international conference on healthcare informatics. IEEE, 2015, pp. 380-389.

[20] M. Legrand, N. Jarrassé, and G. Morel, "Controlling robotic assistive devices with natural body compensations," in ICRA 2020 workshop on Integrating Multidisciplinary Approaches to Advance pHRI, 2020, p. 2 